\title{
Video Recording and Photography with a GoPro Hero 4+ by Surgeon's Hand
}

\author{
A. Sánchez Balado ${ }^{1}$
}

Received: 11 March 2019 / Accepted: 6 August 2019 / Published online: 13 August 2019

(C) Association of Surgeons of India 2019

\section{Dear Sir,}

Intraoperative photography and video recording has become a new important tool in plastic surgery for learning, analysis, teaching residents and fellowships, and its use for congress and courses [1]. Nowadays we have different alternatives such as head-mounted devices, Google Glass or different glasses with an integrated camera, lamp-integrated cameras or cameras attached to surgical lamps [2,3]. Although different authors have struggled to find the best approach to obtain images, both using different camera settings and trying different locations as we mention before, none of them has found a way to manipulate the camera as if we were doing just normal photography out of the surgical theatre. Adjusting working distance in head-mounted cameras is difficult to solve without making the intervention less comfortable for the surgeon. In lamp-theatre integrated cameras, the high distance from the lamp to surgical field can be solved by using high zoom cameras or different lenses compatible with action cameras, which can be expensive.

We found a new approach using GoPro Hero 4+ (GoPro, Inc., San Mateo, CA, USA), introducing it into a transparent sterile surgical bag, which will not interfere with the lens of the camera. This can be done by the surgeon prior surgical hand washing, so the camera is ready to record after bag closure. The surgeon is able to use this high-resolution camera with his/her own hands within the surgical field to obtain better focus and better details of the surgery, as it may be needed in microsurgery training and other interventions performed in a deep surgical field [3]. We also can modify the settings of the camera during different stages of the surgery in order to achieve the best quality images, making the process

A. Sánchez Balado

adrian.sanchez.balado@gmail.com

1 Plastic Reconstructive Surgery Department and Burn Care Unit of Hospital General Universitario de Alicante, C/ Pintor Baeza 10, Alicante, Spain much more versatile. Also with the app, the surgical team is allowed to follow the surgery or visualize different details while streaming. This could be useful for residents to realise the steps of the surgery when surgical field is limited. Finally, intermittent use can also make it a good option for surgeries lasting more than $200 \mathrm{~min}$, which is usually common in microsurgery or head and neck interventions.

We have been using this method for 3 months to record surgical details without problems. We recommend to use disposable bags and close it with an adherent sticker from surgical field after folding the bag, so it will keep its sterility. We also think this is much more affordable compared with other options.

\section{Compliance with Ethical Standards}

Conflict of Interest The author declares that he has no conflict of interest.

Ethical Approval All procedures performed in studies involving human participants were in accordance with the ethical standards of the institutional and/or national research committee and with the 1964 Helsinki declaration and its later amendments or comparable ethical standards.

Informed Consent Informed consent was obtained from all individuals participants included in the study.

\section{References}

1. Bizzotto N, Sandri A, Lavini F, Dall'Oca C, Regis D (2014) Video in operating room: GoPro HERO3 camera on surgeon's head to film operations: a test. Surg Innov 21(3):338e40

2. Graves SN, Shenaq DS, Langerman AJ, Song DH (2015) Video capture of plastic surgery procedures using the GoPro HERO $3+$. Plast Reconstr Surg Glob Open 3(2):e312

3. Zoltie T, Ho M (2018) Viability of a modified GoPro for professional surgical videography. J Plast Reconstr Aesthet Surg 71:1216-1230. https://doi.org/10.1016/j.bjps.2018.05.047

Publisher's Note Springer Nature remains neutral with regard to jurisdictional claims in published maps and institutional affiliations. 